Journal of Bangladesh Academy of Sciences
Journal homepage: http://www.bas.org.bd/publications/jbas.html

Research Article

\title{
Mathematically forecasting for generalized business by using fuzzy trapezoidal numbers
}

Fatema Khatun* and Md. Sahadat Hossain

Department of Mathematics, University of Rajshahi, Rajshahi, Bangladesh

\section{ARTICLE INFO}

\section{Article History}

Received: 16 March 2021

Revised: 22 November 2021

Accepted: 23 November 2021

Keywords: Fuzzy set, Fuzzy triangular number, Fuzzy trapezoidal number, Fuzzy

Delphi Method.

\begin{abstract}
In this paper, we forecasted the future of a business by using fuzzy trapezoidal numbers and the fuzzy Delphi method. This result is compared with another result obtained using the fuzzy triangular numbers and Delphi method. At last, we see that our method is more general than others.
\end{abstract}

\section{Introduction}

The main theme of these Fuzzy trapezoidal numbers defined by Abbasbandy and Hajjari (2010) model construction is to predict the time duration for future forecasting of a business basis on the past historical observation, which is introduced by Ali et al. (2016) and Mutalib et al. (2018). To solve the future forecasting problem of a business, such a way is an appropriate way based on the fuzzy set theory defined by Zadeh (1996 and 1965). Based on the fuzzy set theory, many future forecasting models have been established for businesses using the fuzzy Delphi method introduced by Kuo and Chen (2008).

In this paper, we propose a trapezoidal model based on the fuzzy number and fuzzy Delphi method. Fuzzy numbers are introduced by Bellman and Zadeh (1970) and Kaufmann and Gupta (1985 and 1988). And Delphi method was developed by Roy and Garai (2012). California in the 1940s.

\section{Preliminaries}

2.1 Fuzzy set: A fuzzy set $\mathrm{A}$ is defined by a set, $\mathrm{A}=\left\{\left(x, \mu_{A}(x) \mid x \in A, \mu_{A}(x) \in[0,1]\right\}\right.$, where $\mu_{A}(x)$ is a membership function belonging to $[0,1]$ (Mohanpriya and Jeyanthi, 2016)
2.2 Fuzzy number: A fuzzy number is defined as a convex and normalized fuzzy set on the universe $\mathrm{R}$ (Mohanpriya and Jeyanthi, 2016).

2.3 Triangular fuzzy number: A triangular fuzzy number A with membership function $\mu_{A}(x)$ is defined on $\mathrm{R}$ by (Gani \& Assarudeen, 2012)

$\mathrm{A} \triangleq \mu_{A}(x)=\left\{\begin{array}{cc}\frac{x-a_{1}}{a_{M}-a_{1}} & \text { for } a_{1} \leq x \leq a_{M} \\ \frac{x-a_{2}}{a_{M}-a_{2}} & \text { for } a_{M} \leq x \leq a_{2} \\ 0 & \text { otherwise. }\end{array}\right.$

2.4 Trapezoidal fuzzy number: A trapezoidal fuzzy number A with membership function $\mu_{A}(x)$ is defined on $\mathrm{R}$ by (Mohanpriya and Jeyanthi, 2016).

$\mathrm{A} \triangleq \mu_{A}(x)= \begin{cases}\frac{x-a_{1}}{b_{1}-a_{1}} & \text { for } a_{1} \leq x \leq b_{1} \\ 1 & \text { for } b_{1} \leq x \leq b_{2} \\ \frac{x-a_{2}}{b_{2}-a_{2}} & \text { for } b_{2} \leq x \leq a_{2} \\ 0 & \text { otherwise. }\end{cases}$

2.5 Fuzzy averaging: (i). Triangular fuzzy average formula (Bojadziev and Bojadziev, 2007). Consider $\mathrm{n}$ triangular numbers

$A_{i}=\left(a_{1}^{(i)}, a_{M}^{(i)}, a_{2}^{(i)}\right)$,

where $i=1,2, \ldots \ldots . n$.

The triangular average $A_{\text {ave }}$,

$A_{\text {ave }}=\left(m_{1}, m_{M}, m_{2}\right)=\frac{A_{1}+A_{2}+\cdots+A_{n}}{n}$ 


$$
\begin{aligned}
& =\frac{\left(a_{1}^{(1)}, a_{M}^{(1)}, a_{2}^{(1)}\right)+\cdots+\left(a_{1}^{(n)}, a_{M}^{(n)}, a_{2}^{(n)}\right)}{n} \\
& =\frac{\left(\sum_{i=1}^{n} a_{1}^{(i)}, \sum_{i=1}^{n} a_{M}^{(i)}, \sum_{i=1}^{n} a_{2}^{(i)}\right)}{n}
\end{aligned}
$$

(ii). Trapezoidal fuzzy average formula (Mutalib et al., 2018)

Consider $n$ trapezoidal numbers

$A_{i}=\left(a_{1}^{(i)}, b_{1}^{(i)}, b_{2}^{(i)}, a_{2}^{(i)}\right)$

$$
\text { Where } i=1,2, \ldots \ldots . n \text {. }
$$

The trapezoidal average $A_{\text {ave }}$,

$$
\begin{aligned}
& A_{a v e}=\left(m_{1}, m_{M 1}, m_{M 2}, m_{2}\right)=\frac{A_{1}+A_{2}+\cdots+A_{n}}{n} \\
& =\frac{\left(a_{1}^{(1)}, b_{1}^{(1)}, b_{2}^{(1)}, a_{2}^{(1)}\right)+\cdots+\left(a_{1}^{(n)}, b_{1}^{(n)}, b_{2}^{(n)}, a_{2}^{(n)}\right)}{n} \\
& =\frac{\left(\sum_{i=1}^{n} a_{1}^{(i)}, \sum_{i=1}^{n} b_{1}^{(i)}, \sum_{i=1}^{n} b_{2}^{(i)}, \sum_{i=1}^{n} a_{2}^{(i)}\right)}{n}
\end{aligned}
$$

\section{Related work}

The classical method is generalized by the fuzzy Delphi method for long-range forecasting in management is known as the Delphi method (Milkovich et al. (1972) and Bojadziev and Bojadziev (2007). It can be described as follows:

Expert's responses are analyzed statistically in each round using Fuzzy numbers. A fuzzy statistical analysis is done to find out the difference between individual and mean values obtained from all experts and is communicated to experts for review. Experts' reviews are analyzed, and this process is repeated until the outcome converges to a reasonable solution.

In 1988, Kaufman and Gupta introduced the fuzzy Delphi method.

It consists of the following parts for the triangle (Bojadziev \& Bojadziev, 2007):

Step 1: Experts $E_{i}, i=1, \ldots, n$, are asked to provide the possible realization dates of a certain event in business. The earliest date $a_{1}^{(i)}$, the most plausible date $a_{M}^{(i)}$, and the latest date $a_{2}^{(i)}$. The data given by the experts $E_{i}$ are presented in the form of triangular numbers

$$
\begin{aligned}
& A_{i}=\left(a_{1}^{(i)}, a_{M}^{(i)}, a_{2}^{(i)}\right) \\
& \text { Where } i=1,2, \ldots, n .
\end{aligned}
$$

Step 2: First, the average (mean) $A_{\text {ave }}=$ $\left(m_{1}, m_{M}, m_{2}\right)$ of all $A_{i}$ is computed (see 2.1). Then for each expert $E_{i}$ the deviation between $A_{\text {ave }}$ and $A_{i}$ is computed. It is a triangular number defined by

$$
\begin{aligned}
& A_{\text {ave }}-A_{i}=\left(m_{1}-a_{1}^{(i)}, m_{M}-a_{M}^{(i)}, m_{2}-a_{2}^{(i)}\right) \\
& =\quad\left(\frac{1}{n} \sum_{i=1}^{n} a_{1}^{(i)}-a_{1}^{(i)}, \frac{1}{n} \sum_{i=1}^{n} a_{M}^{(i)}-a_{M}^{(i)},\right. \\
& \left.\frac{1}{n} \sum_{i=1}^{n} a_{2}^{(i)}-a_{2}^{(i)}\right)
\end{aligned}
$$

The deviation $A_{\text {ave }}-A_{i}$ is sent back to the expert $E_{i}$ for reexamination.

Step 3: Each expert $E_{i}$ presents a new triangular number

$B_{i}=\left(b_{1}^{(i)}, b_{M}^{(i)}, b_{2}^{(i)}\right), \quad i=1, \ldots, n$.

This process starts with Step 2 is repeated. The triangular average $B_{\text {ave }}$ is calculated according to formula (2.1) with the difference that now $a_{1}^{(i)}, a_{M}^{(i)}, a_{2}^{(i)}$ are substituted correspondingly by $b_{1}^{(i)}, b_{M}^{(i)}, b_{2}^{(i)}$. If necessary, new triangular numbers $C_{i}=\left(c_{1}^{(i)}, c_{M}^{(i)}, c_{2}^{(i)}\right)$ are generated, and their average $C_{i}$ is calculated. The process could be repeated again and again until two successive means $A_{\text {ave }}, B_{\text {ave }}$, $C_{\text {ave }}$, . . become reasonably close.

Step 4: Later, the same process may be reexamine the forecasting if there is important information available due to new discoveries.

An Innovative Product Time Estimation for Technical Realization (Bojadziev and Bojadziev, 2007).

A group of 15 computer experts are asked to estimate using the Fuzzy Delphi method for the technical realization of a brand-new product, say a cognitive information processing computer. They are ranked equally; hence their opinions carry the same weight. The triangular numbers $A_{i}, i=1, \ldots, 15$ (see (3.1)) presented by the experts are shown in Table 1. 
Khatun and Hossain/J. Bangladesh Acad. Sci. 45(2); 147-154: December 2021

Table 1. Triangular numbers $\boldsymbol{A}_{i}$ presented by experts (first request) (Bojadziev and Bojadziev, 2007).

\begin{tabular}{ccccc}
\hline$E_{\boldsymbol{i}}$ & $\boldsymbol{A}_{\boldsymbol{i}}$ & $\begin{array}{c}\text { Earliest } \\
\text { date }\end{array}$ & $\begin{array}{c}\text { Most } \\
\text { plausible } \\
\text { date }\end{array}$ & $\begin{array}{c}\text { Latest } \\
\text { date }\end{array}$ \\
\hline$E_{1}$ & $A_{1}$ & $a_{1}^{(1)}=1995$ & $a_{M}^{(1)}=2003$ & $a_{2}^{(1)}=2020$ \\
$E_{2}$ & $A_{2}$ & $a_{1}^{(2)}=1997$ & $a_{M}^{(2)}=2004$ & $a_{2}^{(2)}=2010$ \\
$E_{3}$ & $A_{3}$ & $a_{1}^{(3)}=2000$ & $a_{M}^{(3)}=2005$ & $a_{2}^{(3)}=2010$ \\
$E_{4}$ & $A_{4}$ & $a_{1}^{(4)}=1998$ & $a_{M}^{(4)}=2003$ & $a_{2}^{(4)}=2008$ \\
$E_{5}$ & $A_{5}$ & $a_{1}^{(5)}=2000$ & $a_{M}^{(5)}=2005$ & $a_{2}^{(5)}=2015$ \\
$E_{6}$ & $A_{6}$ & $a_{1}^{(6)}=1995$ & $a_{M}^{(6)}=2010$ & $a_{2}^{(6)}=2015$ \\
$E_{7}$ & $A_{7}$ & $a_{1}^{(7)}=2010$ & $a_{M}^{(7)}=2018$ & $a_{2}^{(7)}=2015$ \\
$E_{8}$ & $A_{8}$ & $a_{1}^{(8)}=1995$ & $a_{M}^{(8)}=2007$ & $a_{2}^{(8)}=2013$ \\
$E_{9}$ & $A_{9}$ & $a_{1}^{(9)}=1995$ & $a_{M}^{(9)}=2002$ & $a_{2}^{(9)}=2007$ \\
$E_{10}$ & $A_{10}$ & $a_{1}^{(10)}=2008$ & $a_{M}^{(10)}=2009$ & $a_{2}^{(10)}=2020$ \\
$E_{11}$ & $A_{11}$ & $a_{1}^{(11)}=2010$ & $a_{M}^{(11)}=2020$ & $a_{2}^{(11)}=2024$ \\
$E_{12}$ & $A_{12}$ & $a_{1}^{(12)}=1996$ & $a_{M}^{(12)}=2002$ & $a_{2}^{(12)}=2006$ \\
$E_{13}$ & $A_{13}$ & $a_{1}^{(13)}=1998$ & $a_{M}^{(13)}=2006$ & $a_{2}^{(13)}=2010$ \\
$E_{14}$ & $A_{14}$ & $a_{1}^{(14)}=1997$ & $a_{M}^{(14)}=2005$ & $a_{2}^{(14)}=2012$ \\
$E_{15}$ & $A_{15}$ & $a_{1}^{(15)}=2002$ & $a_{M}^{(15)}=2010$ & $a_{2}^{(15)}=2020$ \\
\hline & & & &
\end{tabular}

To find the average $A_{\text {ave }}$ the sums of the numbers in the last three columns are calculated

$$
\begin{aligned}
& \sum_{i=1}^{15} a_{1}^{(i)}=29996, \sum_{i=1}^{15}=30109 \\
& \sum_{i=1}^{15} a_{2}^{(i)}=30210
\end{aligned}
$$

and substituted into (2.1), which gives

$A_{\text {ave }}=\left(\frac{29996}{15}, \frac{30109}{15}, \frac{30210}{15}\right)=(1999.7,2007.3$, 2014)

or approximately, $A_{\text {ave }}^{a}=(2000,2007,2014)$.
The deviations (3.2) between $A_{\text {ave }}^{a}$ and $A_{i}$ are presented in Table 2 .

Table 2. Deviation $A_{\text {ave }}-A_{i}$.

\begin{tabular}{cccc}
\hline $\boldsymbol{E}_{\boldsymbol{i}}$ & $\boldsymbol{m}_{\mathbf{1}}-\boldsymbol{a}_{\mathbf{1}}^{(i)}$ & $\boldsymbol{m}_{\boldsymbol{M}}-\boldsymbol{a}_{\boldsymbol{M}}^{(i)}$ & $\boldsymbol{m}_{\mathbf{2}}-\boldsymbol{a}_{\mathbf{2}}^{(i)}$ \\
\hline$E_{1}$ & 5 & 4 & -6 \\
$E_{2}$ & 3 & 3 & 4 \\
$E_{3}$ & 0 & 2 & 4 \\
$E_{4}$ & 2 & 4 & 6 \\
$E_{5}$ & 0 & 2 & -1 \\
$E_{6}$ & 5 & -3 & -1 \\
$E_{7}$ & -10 & -11 & -6 \\
$E_{8}$ & 5 & 0 & 1 \\
$E_{9}$ & 5 & 5 & 7 \\
$E_{10}$ & -8 & -2 & -6 \\
$E_{11}$ & -10 & -13 & -10 \\
$E_{12}$ & 4 & 5 & 8 \\
$E_{13}$ & 2 & 1 & 4 \\
$E_{14}$ & 3 & 2 & 2 \\
$E_{15}$ & -2 & -3 & -6 \\
\hline
\end{tabular}

Table 2. shows the divergence of each expert's opinion from the average. A quick glance gives that the experts $E_{3}, E_{5}, E_{8}, E_{13}, E_{14}$ are close to the average while $E_{7}, E_{11}$ is not.

Since the word close is fuzzy, a more detailed study requires some clarification. It can be based on distance $d_{i j}$ between two triangular numbers $A_{i}$ and $A_{j}$. If all $d_{i j}$ are calculated and recorded in a table (in our case consisting of 15 rows and columns), we will have a better grasp of how close various pairs of $A_{i}$ and $A_{j}$ are. Here we do not give a formula for calculating the distance $d_{i j}$ (there are several), 4 but refer to Kaufmann and Gupta (1988).

Suppose the manager is not satisfied with the average $(2000,2007,2014)$. Then the deviation $\left(m_{1}-a_{1}^{(i)}, m_{M}-a_{M}^{(i)}, m_{2}-a_{2}^{(i)}\right)$ is given to each expert $E_{i}$ for reconsideration. The experts 
suggest new triangular numbers $B_{i}$ (see (3.3)) presented in Table 3.

Table 3. Triangular numbers presented by experts (second request) (Bojadziev and Bojadziev, 2007).

\begin{tabular}{ccccc}
\hline$E_{i}$ & $B_{i}$ & Earliest date & $\begin{array}{c}\text { Most plausible } \\
\text { date }\end{array}$ & Latest date \\
\hline$E_{1}$ & $B_{1}$ & $b_{1}^{(1)}=1996$ & $b_{M}^{(1)}=2004$ & $b_{2}^{(1)}=2018$ \\
$E_{2}$ & $B_{2}$ & $b_{1}^{(2)}=1997$ & $b_{M}^{(2)}=2004$ & $b_{2}^{(2)}=2011$ \\
$E_{3}$ & $B_{3}$ & $b_{1}^{(3)}=2000$ & $b_{M}^{(3)}=2005$ & $b_{2}^{(3)}=2011$ \\
$E_{4}$ & $B_{4}$ & $b_{1}^{(4)}=1998$ & $b_{M}^{(4)}=2003$ & $b_{2}^{(4)}=2010$ \\
$E_{5}$ & $B_{5}$ & $b_{1}^{(5)}=2000$ & $b_{M}^{(5)}=2005$ & $b_{2}^{(5)}=2015$ \\
$E_{6}$ & $B_{6}$ & $b_{1}^{(6)}=1997$ & $b_{M}^{(6)}=2009$ & $b_{2}^{(6)}=2015$ \\
$E_{7}$ & $B_{7}$ & $b_{1}^{(7)}=2005$ & $b_{M}^{(7)}=2015$ & $b_{2}^{(7)}=2016$ \\
$E_{8}$ & $B_{8}$ & $b_{1}^{(8)}=1996$ & $b_{M}^{(8)}=2007$ & $b_{2}^{(8)}=2013$ \\
$E_{9}$ & $B_{9}$ & $b_{1}^{(9)}=1997$ & $b_{M}^{(9)}=2004$ & $b_{2}^{(9)}=2010$ \\
$E_{10}$ & $B_{10}$ & $b_{1}^{(10)}=2004$ & $b_{M}^{(10)}=2009$ & $b_{2}^{(10)}=2017$ \\
$E_{11}$ & $B_{11}$ & $b_{1}^{(11)}=2004$ & $b_{M}^{(11)}=2015$ & $b_{2}^{(11)}=2016$ \\
$E_{12}$ & $B_{12}$ & $b_{1}^{(12)}=1996$ & $b_{M}^{(12)}=2004$ & $b_{2}^{(12)}=2006$ \\
$E_{13}$ & $B_{13}$ & $b_{1}^{(13)}=1998$ & $b_{M}^{(13)}=2006$ & $b_{2}^{(13)}=2010$ \\
$E_{14}$ & $B_{14}$ & $b_{1}^{(14)}=1997$ & $b_{M}^{(14)}=2004$ & $b_{2}^{(14)}=2012$ \\
$E_{15}$ & $B_{15}$ & $b_{1}^{(15)}=2001$ & $b_{M}^{(15)}=2009$ & $b_{2}^{(15)}=2015$ \\
\hline & & &
\end{tabular}

The experts $E_{5}, E_{12}$, and $E_{13}$ have not changed their first estimate. Other experts, for instance, $E_{2}, E_{3}, E_{8}$, $E_{14}$, made minimal changes. Using again (2.1), this time to find $B_{\text {ave }}$, gives

$B_{\text {ave }}=(1999.07,2006.9,2013.2)$

Which is approximately,

$B_{a v e}=(1999,2007,2013)$.
The manager is satisfied that $A_{\text {ave }}$ and $B_{\text {ave }}$, also $A_{\text {ave }}^{a}$ and $B_{\text {ave }}^{a}$, are very close (see Fig. 1), stop the fuzzy Delphi process, and accepts the triangular number $B_{\text {ave }}^{a}$ as a combined conclusion of experts' opinions. The interpretation is that the realization of the invention will occur in the time interval [1999, 2013], the supporting interval of the triangular number $B_{\text {ave }}^{a}$ which is almost in central form.

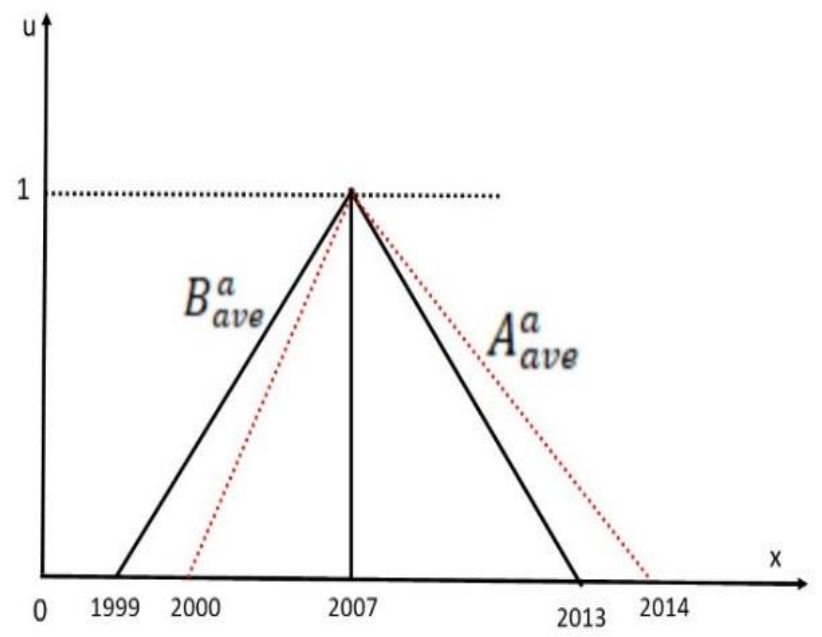

Fig. 1. Average triangular numbers $A_{a v e}^{a}$ and $B_{a v e}^{a}$.

\section{Materials and methods}

The fuzzy Delphi method consists of the following parts for trapezoidal:

Step 1. Experts $E_{i}, \quad i=1, \ldots, n$, are asked to provide the possible realization dates of a particular event in science, technology, or business, namely: the earliest date $a_{1}^{(i)}$, the earliest most plausible date $a_{M 1}^{(i)}$, the latest most plausible date $a_{M 2}^{(i)}$, and the latest date $a_{2}^{(i)}$. The data given by the experts $E_{i}$ are presented in the form of trapezoidal numbers

$A_{i}=\left(a_{1}^{(i)}, a_{M 1}^{(i)}, a_{M 2}^{(i)}, a_{2}^{(i)}\right)$,

Where $i=1,2, \ldots, n$.

Step 2. First, the average (mean) $A_{\text {ave }}=\left(m_{1}, m_{M 1}, m_{M 2}, m_{2}\right)$ of all $A_{i}$ is computed (see 2.2). Then for each expert $E_{i}$ the deviation 
between $A_{\text {ave }}$ and $A_{i}$ is computed. It is a trapezoidal number defined by

$A_{\text {ave }}-A_{i}=\left(m_{1}-a_{1}^{(i)}, m_{M 1}-a_{M 1}^{(i)}, m_{M 2}-\right.$ $\left.a_{M 2}^{(i)}, m_{2}-a_{2}^{(i)}\right)$

$=\left(\frac{1}{n} \sum_{i=1}^{n} a_{1}^{(i)}-a_{1}^{(i)}, \frac{1}{n} \sum_{i=1}^{n} a_{M 1}^{(i)}-a_{M 1}^{(i)}\right.$,

$\left.\frac{1}{n} \sum_{i=1}^{n} a_{M 2}^{(i)}-a_{M 2}^{(i)}, \frac{1}{n} \sum_{i=1}^{n} a_{2}^{(i)}-a_{2}^{(i)}\right)$

The deviation $A_{\text {ave }}-A_{i}$ is sent back to the expert $E_{i}$ for reexamination.

Step 3. Each expert $E_{i}$ presents a new trapezoidal number

$B_{i}=\left(b_{1}^{(i)}, b_{M 1}^{(i)}, b_{M 2}^{(i)}, b_{2}^{(i)}\right), i=1, \ldots, n$.

This process starts with Step 2 is repeated. The trapezoidal average $B_{\text {ave }}$ is calculated according to formula (2.2) with the difference that now $a_{1}^{(i)}, a_{M 1}^{(i)}$, $a_{M 2}^{(i)}, a_{2}^{(i)}$ are substituted correspondingly by $b_{1}^{(i)}, b_{M 1}^{(i)}, b_{M 2}^{(i)}, b_{2}^{(i)}$. If necessary, new trapezoidal numbers $C_{i}=\left(c_{1}^{(i)}, c_{M 1}^{(i)}, c_{M 2}^{(i)}, c_{2}^{(i)}\right)$ are generated, and their average $C_{i}$ is calculated. The process could be repeated again and again until two successive means $A_{\text {ave }}, B_{\text {ave }}, C_{\text {ave }}, \ldots$. become reasonably close.

Step 4. Later, the forecasting may be reexamined by the same process if there is important information available due to new discoveries.

An Innovative Product Time Estimation for Technical Realization.

A group of 15 computer experts are asked to estimate using the Fuzzy Delphi method for the technical realization of a brand-new product, say a cognitive information processing computer. They are ranked equally, hence their opinions carry the same weight. The trapezoidal numbers, $A_{i}, i=1, \ldots, 15$ (see (4.1)) presented by the experts are shown in Table 4 .
Table 4. Trapezoidal numbers $A_{i}$ presented by experts (first request).

\begin{tabular}{|c|c|c|c|c|c|}
\hline$E_{i}$ & $A_{i}$ & $\begin{array}{c}\text { Earliest } \\
\text { date }\end{array}$ & $\begin{array}{c}\text { Earliest } \\
\text { Most } \\
\text { plausible } \\
\text { date }\end{array}$ & $\begin{array}{l}\text { Latest } \\
\text { Most } \\
\text { plausibl } \\
\text { e date }\end{array}$ & $\begin{array}{l}\text { Latest } \\
\text { date }\end{array}$ \\
\hline$E_{1}$ & $A_{1}$ & $a_{1}^{(1)}=1995$ & $\begin{array}{l}a_{M 1}^{(1)} \\
=2003\end{array}$ & $\begin{array}{l}a_{M 2}^{(1)} \\
=2006\end{array}$ & $a_{2}^{(1)}=2020$ \\
\hline$E_{2}$ & $A_{2}$ & $a_{1}^{(2)}=1997$ & $\begin{array}{l}a_{M 1}^{(2)} \\
=2004\end{array}$ & $\begin{array}{l}a_{M 2}^{(2)} \\
=2005\end{array}$ & $a_{2}^{(2)}=2010$ \\
\hline$E_{3}$ & $A_{3}$ & $a_{1}^{(3)}=2000$ & $\begin{array}{l}a_{M 1}^{(3)} \\
=2005\end{array}$ & $\begin{array}{l}a_{M 2}^{(3)} \\
=2007\end{array}$ & $a_{2}^{(3)}=2010$ \\
\hline$E_{4}$ & $A_{4}$ & $a_{1}^{(4)}=1998$ & $\begin{array}{l}a_{M 1}^{(4)} \\
=2003\end{array}$ & $\begin{array}{l}a_{M 2}^{(4)} \\
=2005\end{array}$ & $a_{2}^{(4)}=2008$ \\
\hline$E_{5}$ & $A_{5}$ & $a_{1}^{(5)}=2000$ & $\begin{array}{l}a_{M 1}^{(5)} \\
=2005\end{array}$ & $\begin{array}{l}a_{M 2}^{(5)} \\
=2008\end{array}$ & $a_{2}^{(5)}=2015$ \\
\hline$E_{6}$ & $A_{6}$ & $a_{1}^{(6)}=1995$ & $\begin{array}{l}a_{M 1}^{(6)} \\
=2010\end{array}$ & $\begin{array}{l}a_{M 2}^{(6)} \\
=2012\end{array}$ & $a_{2}^{(6)}=2015$ \\
\hline$E_{7}$ & $A_{7}$ & $a_{1}^{(7)}=2010$ & $\begin{array}{l}a_{M 1}^{(7)} \\
=2018\end{array}$ & $\begin{array}{l}a_{M 2}^{(7)} \\
=2019\end{array}$ & $a_{2}^{(7)}=2015$ \\
\hline$E_{8}$ & $A_{8}$ & $a_{1}^{(8)}=1995$ & $\begin{array}{l}a_{M 1}^{(8)} \\
=2007\end{array}$ & $\begin{array}{l}a_{M 2}^{(8)} \\
=2010\end{array}$ & $a_{2}^{(8)}=2013$ \\
\hline$E_{9}$ & $A_{9}$ & $a_{1}^{(9)}=1995$ & $\begin{array}{l}a_{M 1}^{(9)} \\
=2002\end{array}$ & $\begin{array}{l}a_{M 2}^{(9)} \\
=2005\end{array}$ & $a_{2}^{(9)}=2007$ \\
\hline$E_{10}$ & $A_{10}$ & $a_{1}^{(10)}=2008$ & $\begin{array}{l}a_{M 1}^{(10)} \\
=2009\end{array}$ & $\begin{array}{l}a_{M 2}^{(10)} \\
=2013\end{array}$ & $a_{2}^{(10)}=2020$ \\
\hline$E_{11}$ & $A_{11}$ & $a_{1}^{(11)}=2010$ & $\begin{array}{l}a_{M 1}^{(11)} \\
=2020\end{array}$ & $\begin{array}{l}a_{M 2}^{(11)} \\
=2022\end{array}$ & $a_{2}^{(11)}=2024$ \\
\hline$E_{12}$ & $A_{12}$ & $a_{1}^{(12)}=1996$ & $\begin{array}{l}a_{M 1}^{(12)} \\
=2002\end{array}$ & $\begin{array}{l}a_{M 2}^{(12)} \\
=2003\end{array}$ & $a_{2}^{(12)}=2006$ \\
\hline$E_{13}$ & $A_{13}$ & $a_{1}^{(13)}=1998$ & $\begin{array}{l}a_{M 1}^{(13)} \\
=2006\end{array}$ & $\begin{array}{l}a_{M 2}^{(13)} \\
=2008\end{array}$ & $a_{2}^{(13)}=2010$ \\
\hline$E_{14}$ & $A_{14}$ & $a_{1}^{(14)}=1997$ & $\begin{array}{l}a_{M 1}^{(14)} \\
=2005\end{array}$ & $\begin{array}{l}a_{M 2}^{(14)} \\
=2008\end{array}$ & $a_{2}^{(14)}=2012$ \\
\hline & $A_{15}$ & $a_{1}^{(15)}=2002$ & $\begin{array}{l}a_{M 1}^{(15)} \\
=2010\end{array}$ & $\begin{array}{l}a_{M 2}^{(15)} \\
=2013\end{array}$ & $a_{2}^{(15)}=2020$ \\
\hline
\end{tabular}


To find the average $A_{\text {ave }}$ the sums of the numbers in the last four columns are calculated

$$
\text { 9996, } \begin{aligned}
\sum_{i=1}^{15} a_{M 1}^{(i)} & =30109, \\
\sum_{i=1}^{15} a_{M 2}^{(i)} & =30144, \sum_{i=1}^{15} a_{2}^{(i)}=30210
\end{aligned}
$$

and substituted into (2.2) which gives

$A_{\text {ave }}=\left(\frac{29996}{15}, \frac{30109}{15}, \frac{30144}{15}, \frac{30210}{15}\right)$

$=(1999.7,2007.3,2009.6,2014)$

or approximately,

$$
A_{\text {ave }}^{a}=(2000,2007,2010,2014) .
$$

The deviations (4.2) between $A_{\text {ave }}^{a}$ and $A_{i}$ are presented in Table 5.

Table 5. Deviation $A_{\text {ave }}-A_{i}$.

\begin{tabular}{ccccc}
\hline $\boldsymbol{E}_{\boldsymbol{i}}$ & $\begin{array}{c}\boldsymbol{m}_{\mathbf{1}} \\
-\boldsymbol{a}_{\mathbf{1}}^{(\boldsymbol{i})}\end{array}$ & $\begin{array}{c}\boldsymbol{m}_{\boldsymbol{M} \mathbf{1}} \\
-\boldsymbol{a}_{\boldsymbol{M 1}}^{(\boldsymbol{i})}\end{array}$ & $\begin{array}{c}\boldsymbol{m}_{\boldsymbol{M} \mathbf{2}} \\
-\boldsymbol{a}_{\boldsymbol{M} \mathbf{2}}^{(i)}\end{array}$ & $\begin{array}{c}\boldsymbol{m}_{\mathbf{2}} \\
-\boldsymbol{a}_{\mathbf{2}}^{(\boldsymbol{i})}\end{array}$ \\
\hline$E_{1}$ & 5 & 4 & 4 & -6 \\
$E_{2}$ & 3 & 3 & 5 & 4 \\
$E_{3}$ & 0 & 2 & 3 & 4 \\
$E_{4}$ & 2 & 4 & 5 & 6 \\
$E_{5}$ & 0 & 2 & 2 & -1 \\
$E_{6}$ & 5 & -3 & -2 & -1 \\
$E_{7}$ & -10 & -11 & -9 & -6 \\
$E_{8}$ & 5 & 0 & 0 & 1 \\
$E_{9}$ & 5 & 5 & 5 & 7 \\
$E_{10}$ & -8 & -2 & -3 & -6 \\
$E_{11}$ & -10 & -13 & -12 & -10 \\
$E_{12}$ & 4 & 5 & 7 & 8 \\
$E_{13}$ & 2 & 1 & 2 & 4 \\
$E_{14}$ & 3 & 2 & 2 & 2 \\
$E_{15}$ & -2 & -3 & -3 & -6 \\
\hline & & & & \\
\hline
\end{tabular}

Table 5. shows the divergence of each expert's opinion from the average. A quick glance gives that the experts $E_{3}, E_{5}, E_{8}, E_{13}, E_{14}$ are close to the average while $E_{7}, E_{11}$ is not.
Since the word close is fuzzy, a more detailed study requires some clarification. It can be based on distance $d_{i j}$ between two trapezoidal numbers $A_{i}$ and $A_{j}$. If all $d_{i j}$ are calculated and recorded in a table (in our case consisting of 15 rows and columns), we will have a better grasp of how close various pairs of $A_{i}$ and $A_{j}$ are. Here we do not give a formula for calculating the distance $d_{i j}$ (there are several), 4 but refer to Kaufmann \& Gupta (1988).

Suppose the manager is not satisfied with the average (2000, 2007, 2010, 2014). Then the deviation $\left(m_{1}-\right.$ $\left.a_{1}^{(i)}, m_{M 1}-a_{M 1}^{(i)}, m_{M 2}-a_{M 2}^{(i)}, m_{2}-a_{2}^{(i)}\right)$ is given to each expert $E_{i}$ for reconsideration. The experts suggest new trapezoidal numbers $B_{i}$ (see (4.3) presented in Table 6.

\begin{tabular}{|c|c|c|c|c|c|}
\hline$E_{i}$ & $B_{i}$ & $\begin{array}{l}\text { Earliest } \\
\text { date }\end{array}$ & $\begin{array}{c}\text { Earliest } \\
\text { Most } \\
\text { plausible } \\
\text { date }\end{array}$ & $\begin{array}{c}\text { Latest } \\
\text { Most } \\
\text { plausible } \\
\text { date }\end{array}$ & $\begin{array}{c}\text { Latest } \\
\text { date }\end{array}$ \\
\hline$E_{1}$ & $B_{1}$ & $\begin{array}{l}b_{1}^{(1)} \\
=1996\end{array}$ & $\begin{array}{l}b_{M 1}^{(1)} \\
=2004\end{array}$ & $\begin{array}{l}b_{M 2}^{(1)} \\
=2007\end{array}$ & $\begin{array}{l}b_{2}^{(1)} \\
=2018\end{array}$ \\
\hline$E_{2}$ & $B_{2}$ & $\begin{array}{l}b_{1}^{(2)} \\
=1997\end{array}$ & $\begin{array}{l}b_{M 1}^{(2)} \\
=2004\end{array}$ & $\begin{array}{l}b_{M 2}^{(2)} \\
=2006\end{array}$ & $\begin{array}{l}b_{2}^{(2)} \\
=2011\end{array}$ \\
\hline$E_{3}$ & $B_{3}$ & $\begin{array}{l}b_{1}^{(3)} \\
=2000\end{array}$ & $\begin{array}{l}b_{M 1}^{(3)} \\
=2005\end{array}$ & $\begin{array}{l}b_{M 2}^{(3)} \\
=2005\end{array}$ & $\begin{array}{l}b_{2}^{(3)} \\
=2011\end{array}$ \\
\hline$E_{4}$ & $B_{4}$ & $\begin{array}{l}b_{1}^{(4)} \\
=1998\end{array}$ & $\begin{array}{l}b_{M 1}^{(4)} \\
=2003\end{array}$ & $\begin{array}{l}b_{M 2}^{(4)} \\
=2005\end{array}$ & $\begin{array}{l}b_{2}^{(4)} \\
=2010\end{array}$ \\
\hline$E_{5}$ & $B_{5}$ & $\begin{array}{l}b_{1}^{(5)} \\
=2000\end{array}$ & $\begin{array}{l}b_{M 1}^{(5)} \\
=2005\end{array}$ & $\begin{array}{l}b_{M 2}^{(5)} \\
=2008\end{array}$ & $\begin{array}{l}b_{2}^{(5)} \\
=2015\end{array}$ \\
\hline$E_{6}$ & $B_{6}$ & $\begin{array}{l}b_{1}^{(6)} \\
=1997\end{array}$ & $\begin{array}{l}b_{M 1}^{(6)} \\
=2009\end{array}$ & $\begin{array}{l}b_{M 2}^{(6)} \\
=2011\end{array}$ & $\begin{array}{l}b_{2}^{(6)} \\
=2015\end{array}$ \\
\hline$E_{7}$ & $B_{7}$ & $\begin{array}{l}b_{1}^{(7)} \\
=2005\end{array}$ & $\begin{array}{l}b_{M 1}^{(7)} \\
=2015\end{array}$ & $\begin{array}{l}b_{M 2}^{(7)} \\
=2016\end{array}$ & $\begin{array}{l}b_{2}^{(7)} \\
=2016\end{array}$ \\
\hline
\end{tabular}

Table 6. Trapezoidal numbers presented by experts (second request). 


\begin{tabular}{|c|c|c|c|c|c|}
\hline \multirow[t]{2}{*}{$E_{8}$} & $B_{8}$ & $b_{1}^{(8)}$ & $b_{M 1}^{(8)}$ & $b_{M 2}^{(8)}$ & $b_{2}^{(8)}$ \\
\hline & & $=1996$ & $=2007$ & $=2010$ & $=2013$ \\
\hline \multirow[t]{2}{*}{$E_{9}$} & $B_{9}$ & $b_{1}^{(9)}$ & $b_{M 1}^{(9)}$ & $b_{M 2}^{(9)}$ & $b_{2}^{(9)}$ \\
\hline & & $=1997$ & $=2004$ & $=2007$ & $=2010$ \\
\hline \multirow[t]{2}{*}{$E_{10}$} & $B_{10}$ & $b_{1}^{(10)}$ & $b_{M 1}^{(10)}$ & $b_{M 2}^{(10)}$ & $b_{2}^{(10)}$ \\
\hline & & $=2004$ & $=2009$ & $=20013$ & $=2017$ \\
\hline \multirow[t]{2}{*}{$E_{11}$} & $B_{11}$ & $b_{1}^{(11)}$ & $b_{M 1}^{(11)}$ & $b_{M 2}^{(11)}$ & $b_{2}^{(11)}$ \\
\hline & & $=2004$ & $=2015$ & $=2017$ & $=2016$ \\
\hline \multirow[t]{2}{*}{$E_{12}$} & $B_{12}$ & $b_{1}^{(12)}$ & $b_{M 1}^{(12)}$ & $b_{M 2}^{(12)}$ & $b_{2}^{(12)}$ \\
\hline & & $=1996$ & $=2004$ & $=2005$ & $=2006$ \\
\hline \multirow[t]{2}{*}{$E_{13}$} & $B_{13}$ & $b_{1}^{(13)}$ & $b_{M 1}^{(13)}$ & $b_{M 2}^{(13)}$ & $b_{2}^{(13)}$ \\
\hline & & $=1998$ & $=2006$ & $=2008$ & $=2010$ \\
\hline \multirow[t]{2}{*}{$E_{14}$} & $B_{14}$ & $b_{1}^{(14)}$ & $b_{M 1}^{(14)}$ & $b_{M 2}^{(14)}$ & $b_{2}^{(14)}$ \\
\hline & & $=1997$ & $=2004$ & $=2007$ & $=2012$ \\
\hline \multirow[t]{2}{*}{$E_{15}$} & $B_{15}$ & $b_{1}^{(15)}$ & $b_{M 1}^{(15)}$ & $b_{M 2}^{(15)}$ & $b_{2}^{(15)}$ \\
\hline & & $=2001$ & $=2009$ & $=2012$ & $=2015$ \\
\hline
\end{tabular}

The experts $E_{5}, E_{12}$, and $E_{13}$ have not changed their first estimate. Other experts, for instance, $E_{2}, E_{3}, E_{8}$, $E_{14}$, made minimal changes. Using again (2.2), this time to find $B_{\text {ave }}$, gives

$$
B_{\text {ave }}=(1999.07,2006.9,2009.13,2013.2)
$$

which is approximately,

$$
B_{\text {ave }}=(1999,2007,2009,2013) \text {. }
$$

The manager is satisfied that $A_{\text {ave }}$ and $B_{\text {ave }}$, also $A_{a v e}^{a}$ and $B_{a v e}^{a}$, are very close (see Fig. 2.), stops the fuzzy Delphi process, and accepts the trapezoidal number $B_{a v e}^{a}$ as a combined conclusion of experts' opinions. The interpretation is that the realization of the invention will occur in the time interval [1999, 2013], the supporting interval of the trapezoidal number $B_{a v e}^{a}$, which is almost in central form.

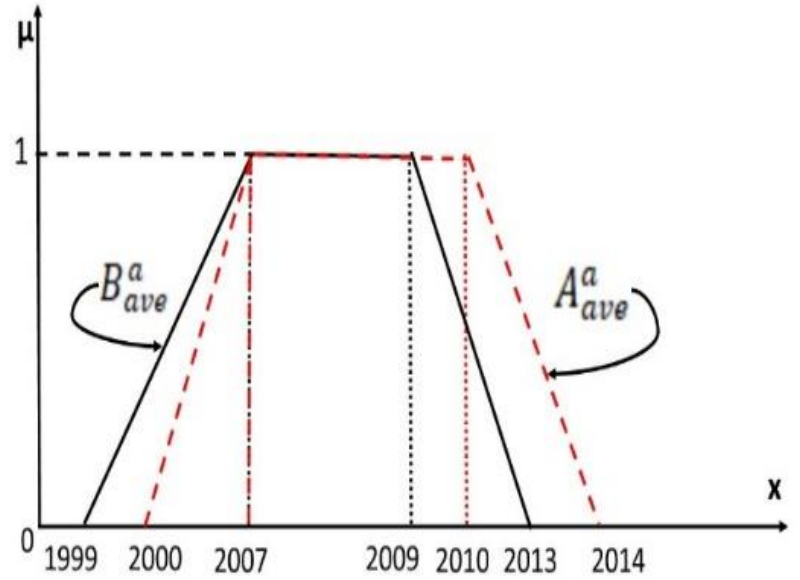

Fig. 2. Average trapezoidal numbers $A_{a v e}^{a}$ and $B_{a v e}^{a}$.

\section{Results and Discussion}

In this article, we use triangular and trapezoidal numbers. By comparing two of these numbers, we get,

(i) From the triangle, we get one peak point from where it's not sure how many days it will run well. On the other hand, we get an interval of the peak points that define that the business will run well in this interval from trapezoidal numbers.

(ii) Also, we see from the trapezoidal numbers figure that a fast business will build up or fall. But there is no proper definition in the triangular numbers.

\section{Conclusion}

Here we see that trapezoidal numbers give better results than triangular numbers for future business forecasting since the trapezoidal numbers are more generalized than triangular numbers. So, the results we have gotten using trapezoidal numbers will be better than triangular numbers.

\section{Conflicts of Interest}

The authors declare that they have no conflicts of interest regarding the publication of this article.

\section{References}

Abbasbandy S and Hajjari T. Weighted trapezoidal approximation-preserving cores of a fuzzy number. Comput. Math. Appl. 2010; 59(9): 3066-3077. 
Ali D, Yohanna M, Puwu MI and Garkida BM. Long-term load forecast modelling using a fuzzy logic approach. Pac. Sci. Rev. A: Nat. Sci. Engineer. 2016; 18(2): 123-127.

Bellman RE and Zadeh LA. Decision-making in a fuzzy environment. Manage. Sci., 1970; 17(4): 141-164.

Bojadziev $G$ and Bojadziev M. Fuzzy logic for business, finance, and management. Advances in Fuzzy Systems Applications and Theory: Volume 23, $2^{\text {nd }}$ ed., World Scientific Singapore, 2007.

Gani AN and Assarudeen SNM A new operation on triangular fuzzy number for solving fuzzy linear programming problem. Appl. Math. Sci., 2012; 6(11): 525-532.

Kaufmann A and Gupta MM. Introduction to fuzzy arithmetic: theory and applications. Electrical Computer Science and Engineering Series. Van Nostr and Reinhold Company, New York, 1985.

Kaufmann A and Gupta MM. Fuzzy mathematical models in engineering and management science. Elsevier Science Inc., New York 1988.

Kuo YF and Chen PC. Constructing performance appraisal indicators for mobility of the service industries using fuzzy Delphi method. Expert Syst. Appl. 2008; 35(4): 1930-1939.
Milkovich GT, Annoni AJ and Mahoney TA. The use of the Delphi procedures in manpower forecasting. Manage. Sci. 1972; 19(4-part-1): 381-388.

Mohanpriya S and Jeyanthi V. Modified procedure to solve fuzzy transshipment problem by using trapezoidal fuzzy number. Int. J. Math. Stat, Inv. 2016; 4: 30-34.

Mutalib SMA, Ramli $\mathrm{N}$ and Mohamad D. Forecasting fuzzy time series model based on trapezoidal fuzzy numbers with area and height similarity measure concept. In AIP Conference Proceedings, AIP Publishing LLC. 2018; 1974(1): 020040.

Roy TK and Garai A. Intuitionistic fuzzy delphi method: more realistic and interactive forecasting tool. NIFS 2012; 18(2): 37-50.

Zadeh LA. Fuzzy sets. Info. Control 1965; 8(3): 338-353.

Zadeh LA. Fuzzy sets, fuzzy logic, and fuzzy systems: selected papers. In: Advances in Fuzzy Systems- Applications and Theory. Volume 6, Eds. Klir GJ and Yuan B. 1996. 